\title{
Sequential Systemic Treatment in Advanced Hepatocellular Carcinoma Is Able to Prolong Median Survival to More than 3 Years in a Selected Real-World Cohort
}

\author{
Johann von Felden ${ }^{a}$ Kathrin Karkmann ${ }^{a}$ Harald Ittrich ${ }^{b}$ Ines Gil-Ibanez ${ }^{a}$ \\ Thorben Fründt $^{a}$ Jenny Krause ${ }^{a}$ Ansgar W. Lohse ${ }^{a}$ Henning Wege ${ }^{a}$ \\ Kornelius Schulze ${ }^{a}$ \\ a. Department of Internal Medicine, University Medical Center Hamburg-Eppendorf, Hamburg, Germany; \\ ${ }^{b}$ Department for Diagnostic and Interventional Radiology and Nuclear Medicine, University Medical Center \\ Hamburg-Eppendorf, Hamburg, Germany
}

\footnotetext{
Keywords

Immunotherapy · HCC · Liver cancer · Immuno-oncology · Targeted therapy
}

\begin{abstract}
Introduction: The number of efficacious systemic agents for advanced hepatocellular carcinoma (HCC) has rapidly increased over the past 3 years. However, guidance for optimal sequential systemic treatment in patients with advanced disease and experience with outcome and safety profiles are lacking. Objective: We aimed to assess efficacy and tolerability of sequential systemic therapy of advanced HCC. Methods: Our single-center study prospectively followed 14 patients who received multiple, sequential systemic therapies after progression or intolerance to sorafenib. Endpoints were overall and progression-free survival (OS, PFS), objective response rate (ORR), and treatment-emergent adverse events (TEAE). Results: Patients had well-compensated liver function and good performance status at start of each systemic therapy. Agents included sorafenib $(n=14)$, regorafenib $(n=10)$, immunotherapy with nivolumab or pembrolizumab $(n=10)$, lenvatinib $(n=3)$, ramucirumab $(n=2)$, and
\end{abstract}

others, with a median of 3 lines of systemic therapy per patient. Median OS was 37.4 months from initiation of first-line therapy with sorafenib. PFS and ORR for sorafenib, regorafenib, and immunotherapy were 6.6, 5.3, and 6.6 months, and $15.4,11.1$, and $22.2 \%$, respectively. TEAE were frequent (46-80\%), but mostly manageable during tyrosine kinase inhibitor therapy and without the need for termination in most patients. However, TEAE due to immunotherapy (60\%) led to cessation of treatment in $40 \%$ of the patients. Conclusions: Sequential systemic therapy is able to prolong median OS in selected patients with advanced HCC to more than 3 years. TEAE are frequent, but manageable, and the quality of adverse events depends on the respective agent. Further investigation of potential predictive biomarkers for treatment allocation is needed.

(c) 2020 S. Karger AG, Basel

\section{Introduction}

Liver cancer ranks fourth among the most frequent causes of cancer-related death [1]. Hepatocellular carcinoma (HCC) accounts for $>90 \%$ of the primary liver 
cancers, and its high mortality is primarily due to its late diagnosis at advanced stages with distant metastases or macrovascular invasion, and secondarily, a result of limited therapeutic options for advanced-stage disease [2-5].

Since the SHARP trial in 2008, sorafenib (SOR) has served as a standard first-line systemic treatment for advanced HCC $[6,7]$. After several years of disappointing failures in phase III clinical trials [3, 8], lenvatinib (LEN), an anti-angiogenic tyrosine kinase inhibitor (TKI), has been demonstrated to be non-inferior to SOR as a firstline treatment (overall survival [OS] 13.6 vs. 12.3 months, hazard ratio $0.92,95 \%$ CI $0.79-1.06$ ), and has been approved by the U.S. Food and Drug Administration (FDA) and the European Medicine Agency (EMA) [9]. Regarding second-line therapy, regorafenib (REG), another multikinase inhibitor, was the first agent tested positive in a phase III clinical trial with benefit in terms of OS after tumor progression on SOR compared to placebo (RESORCE trial; OS 10.6 vs. 7.8 months, hazard ratio $0.63,95 \%$ CI $0.50-0.79$ ) [10]. This study further demonstrated a significant improvement in progression-free survival (PFS), time to progression (TTP), and an objective response rate (ORR) of 11 versus $4 \%$ in the placebo $\operatorname{arm}(p<0.005)$ [10]. With respect to treatment-emergent adverse events (TEAE) grade 3 or 4 , arterial hypertension (15\%), hand-foot syndrome (HFS, 13\%), fatigue (9\%), and diarrhea (3\%) were most frequently observed [10]. Further phase III clinical trials have tested cabozantinib, a MET, VEGFR2, and RET inhibitor, in the CELESTIAL trial [11], and ramucirumab (RAM), a VEGFR2 inhibitory monoclonal antibody, in the REACH-2 trial [12], as being superior to placebo in second-line therapy with a prolonged median OS (the latter in patients with AFP $>400 \mathrm{ng} / \mathrm{mL}$ ). In addition, immune checkpoint inhibition following tumor progression or TEAE on SOR has recently been introduced by the phase I/II CHECKMATE-040 trial [13], in which the PD-1 inhibitor nivolumab (NIVO) has demonstrated promising results with ORR close to $20 \%$ [13]. Nevertheless, $25 \%$ of the patients experienced TEAE grade 3 or 4 , for example, rash, pruritus, signs of hepatitis, or pancreatitis [13]. This has led to accelerated approval in North America by the FDA for second-line treatment. Similarly, another PD-1 antibody, pembrolizumab (PEM), has also received accelerated FDA-approval as a second-line treatment based on similar phase II trial results [14]. Yet, the respective phase III clinical trials testing NIVO versus SOR as a firstline therapy (CHECKMATE-459, NCT02576509) and PEM versus placebo as a second-line treatment (KEYNOTE-240, NCT02702401) have failed to meet their primary endpoints, which suggests that biomarkers are needed to identify patients who benefit from these fairly new agents.
Table 1. Baseline characteristics of the cohort $(n=14)$

$\begin{array}{lc}\begin{array}{l}\text { Mean age (range), years } \\ \text { Male }\end{array} & 66(56-75) \\ \text { Etiology } & 11(78.6) \\ \quad \text { Alcohol related } & 7(50) \\ \text { HBV related } & 1(7.1) \\ \text { HCV related } & 3(21.4) \\ \text { NASH } & 2(14.3) \\ \text { Adenoma } & 1(7.1) \\ \text { Cryptogenic } & 2(14.3) \\ \text { Cirrhosis } & 10(71.4) \\ \text { Previous treatments } & \\ \quad \text { Surgery } & 5(35.7) \\ \text { Locoregional therapy } & 8(57.1) \\ \text { Treatment-naïve } & 5(35.7)\end{array}$

Baseline characteristics of the cohort at start of sequential systemic therapy. Values are presented as $n(\%)$, unless otherwise indicated. HBV, hepatitis B virus; $\mathrm{HCV}$, hepatitis $\mathrm{C}$ virus; $\mathrm{NASH}$, non-alcoholic steatohepatitis. ${ }^{a}$ Two patients had co-etiologies. $\mathrm{b}$ Three patients had multiple previous treatments including surgery and locoregional therapy.

Since the field of systemic therapy for patients with HCC is currently profoundly changing and guidance for effective allocation of patients to available agents is lacking, we aimed to investigate real-life outcomes of sequential systemic treatment. In addition, we were also interested in the safety profile and tolerability of the new agents listed above.

\section{Patients and Methods}

For this mono-center study, all patients who received subsequent systemic therapy for advanced HCC after progression or intolerability to SOR between November 2014 and September 2018 were consecutively enrolled and followed. Diagnosis and staging of HCC was made according to current guidelines [2, 3].

Clinical baseline characteristics such as etiology and stage of liver disease were obtained by review of medical records. Cirrhosis was diagnosed through biopsy or noninvasively, including liver stiffness measurements and/or aspartate transaminase-to-platelet ratio index ( $>2)$. Performance status, as indicated by the Eastern Cooperative Oncology Group (ECOG), and laboratory tests were recorded at initiation and throughout each treatment. Information about TEAE was assessed by physical examination and questionnaires at each visit and graded according to the Common Terminology Criteria for Adverse Events (CTCAE, version 4).

All patients were treated at the I. Department of Medicine, University Medical Center Hamburg-Eppendorf. The choice of treatment was based upon the discretion of the treating physician and available evidence at the time of start of sequential therapy $[10,13]$.

Primary endpoint was OS, calculated from initiation of the first-line systemic therapy (i.e., SOR). Secondary endpoints were PFS and ORR as well as quantity and quality of TEAE for each major systemic agent (i.e., SOR, REG, NIVO/PEM). Tumor response was assessed by mRECIST criteria with contrast-enhanced com- von Felden et al. 
Table 2. Key characteristics of patients at start of each of the main sequential systemic therapies and radiographic response according to mRECIST

\begin{tabular}{|c|c|c|c|}
\hline & $\begin{array}{l}\text { SOR } \\
(n=14)\end{array}$ & $\begin{array}{l}\text { REG } \\
(n=10)\end{array}$ & $\begin{array}{l}\text { NIVO/PEM } \\
(n=10)\end{array}$ \\
\hline \multicolumn{4}{|l|}{ Respective line of treatment } \\
\hline First line & 14 & 0 & 0 \\
\hline Second line & 0 & 7 & 3 \\
\hline$\geq$ Third line & 0 & 3 & 7 \\
\hline Cirrhosis & 10 & 6 & 6 \\
\hline CPT score A & 5 & 5 & 4 \\
\hline CPT score B & 4 & 1 & 2 \\
\hline CPT score C & 1 & 0 & 0 \\
\hline \multicolumn{4}{|l|}{ ECOG } \\
\hline Status $0 / 1$ & 14 & 9 & 10 \\
\hline Status 2 & 0 & 1 & 0 \\
\hline \multicolumn{4}{|l|}{ BCLC } \\
\hline Stage $\mathrm{B}^{\mathrm{a}}$ & 1 & 1 & 0 \\
\hline Stage C & 13 & 9 & 10 \\
\hline Macrovascular invasion ${ }^{\mathrm{b}}$ & 4 & 3 & 5 \\
\hline Extrahepatic spread ${ }^{\mathrm{b}}$ & 10 & 8 & 9 \\
\hline Median AFP (range), ng/mL & $20(2-23,841)$ & $418(2-71,335)$ & $66(4-12,412)$ \\
\hline \multicolumn{4}{|l|}{ Response rates ${ }^{\mathrm{c}}, n(\%)$} \\
\hline Complete response & 0 & 0 & 0 \\
\hline Partial response & $2(15.4)$ & $1(11.1)$ & $2(22.2)$ \\
\hline Stable disease & $4(30.8)$ & $5(55.5)$ & $2(22.2)$ \\
\hline Progressive disease & $7(50)$ & $3(33.3)$ & $5(55.5)$ \\
\hline
\end{tabular}

Categorical and continuous variables are displayed in absolute numbers and median with range, respectively. AFP, alpha-fetoprotein; BCLC, Barcelona Clinic for Liver Cancer classification; CPT, Child-Pugh-Turcotte; NIVO, nivolumab; PEM, pembrolizumab; REG, regorafenib; SOR, sorafenib. ${ }^{\text {a }}$ One patient received systemic therapy after progression of locoregional therapy. ${ }^{\mathrm{b}}$ Some patients had both macrovascular invasion and extrahepatic spread ( $n=1 \mathrm{SOR}, n=2$, REG, $n=4 \mathrm{NIVO} / \mathrm{PEM}) .{ }^{\mathrm{c}}$ One patient with missing data on radiologic response.

puted tomography or magnetic resonance imaging every 8-12 weeks as recommended by current guidelines $[2,3,15]$. We used the STROBE cohort checklist when writing our report [16].

The datasets analyzed in this study are available from the corresponding author upon reasonable request.

\section{Results}

\section{Baseline Characteristics of the Cohort}

We consecutively enrolled 14 patients, who have received sequential systemic therapy after progression or intolerance to SOR. As expected, male gender dominated with $78.6 \%$, and the median age of patients was 66 years (range 56-75) (see Table 1 for baseline characteristics). We additionally summarized key clinical variables at the initiation of each of the major systemic treatments (Table 2).

\section{Efficacy of Systemic Treatments}

All 14 patients started with SOR as the first-line systemic agent. REG and immunotherapy (i.e., NIVO [9 cas- es] or PEM [ 1 case]) were prescribed in 7 and 3 cases for second-line, and in 3 and 7 cases for third-line treatment, respectively. LEN was administered in 3, RAM in 2, capecitabine in 2 , doxorubicin in 1 patient, and 2 patients were treated within the ReLive study with doxorubicin Transdrug (NCT01655693), respectively (Fig. 1a). The cohort reached a median follow-up time of 22.3 months (range 8.3-47.3). Overall, 7 deaths occurred with a median OS of 37.4 months (Fig. 1b). All deaths were due to tumor progression, and none was judged as treatmentrelated by the investigators. Survival rates at 12,24 , and 36 months were $71.4,55.6$, and $55.6 \%$, respectively. The median number of systemic agents per patient was 3 , with a maximum of 5 lines of treatment in 3 patients so far with a follow-up time of $19.9,34.2$, and 42.3 months, respectively. For detailed efficacy analysis, we focused on the main 3 systemic agents in our cohort, that is, SOR $(n=$ $14)$, REG $(n=10)$, and immunotherapy with NIVO/PEM $(n=10)$. Median PFS was 6.6, 5.3, and 6.6 months for SOR, REG, and immunotherapy, respectively (Fig. 1c-e). Of these, immunotherapy had the highest ORR with $22.2 \%$ (Table 2), which is in line with published results 


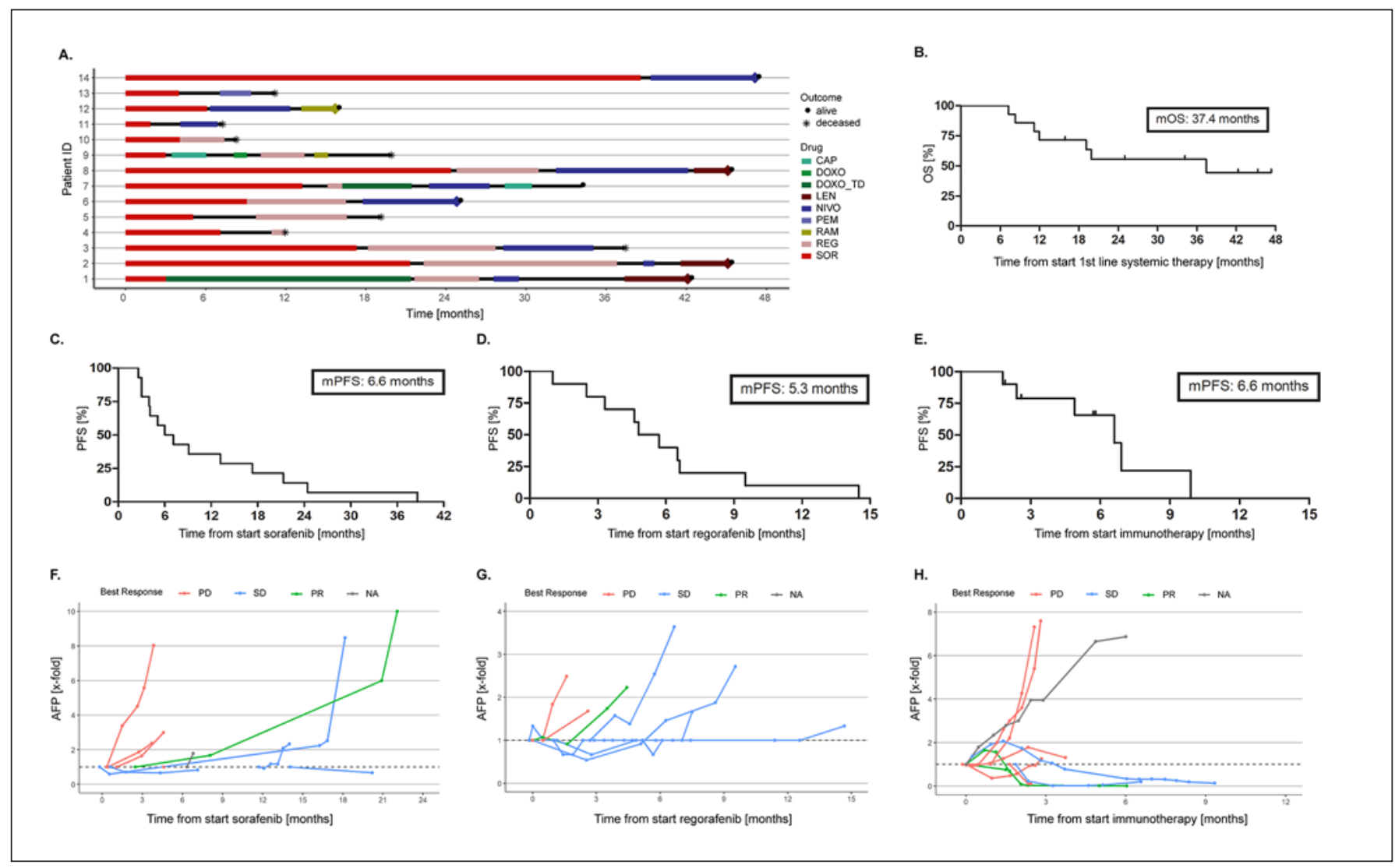

Fig. 1. a Sequential systemic therapies by patient over time. b OS. Seven patients are still alive $(\bullet)$, of whom 6 are currently receiving therapy. c-e Progression-free survival (PFS) for each of the main treatments. $\mathbf{f}-\mathbf{h}$ Course of AFP (x-fold changes) according to best response for treatment with SOR (f), REG (g), and immunotherapy (h). Displayed are $\mathrm{x}$-fold changes in AFP. One patient had missing data on radiologic response (NA). For illustration purposes, data for immunotherapy are limited to a maximum of 8 -fold increase (for uncropped graph, see suppl. Fig. S1). CAP, capecitabine; DOXO(_TD), doxorubicin (Transdrug); LEN, lenvatinib; NIVO, nivolumab; PD, progressive disease; PEM, pembrolizumab; PR, partial response; RAM, ramucirumab; REG, regorafenib; SD, stable disease; SOR, sorafenib.

\section{Treatment-Emergent Adverse Events}

As expected, TEAE occurred frequently. TEAE following SOR or REG were in line with previous reports and consisted mostly of HFS, hypertension, diarrhea, stomatitis, and fatigue. Generally, TEAE were low grade (i.e., CTCAE grade 1 and 2) and manageable following the recommendations for management of TKI AEs. Yet, dose-reduction (but not interruption) was necessary in $50 \%$ of the patients treated with REG, and one death on treatment occurred, which was regarded as unrelated. One patient on SOR was switched to NIVO due to intolerance. Regarding immunotherapy with NIVO or PEM, most TEAE were immune-related, that is, hepatitis, myositis, pneumonitis, and of high grade (i.e., CTCAE grade 3 and 4 ) in $60 \%$ of the cases, leading to termination of immunotherapy in these patients despite treatment with corticosteroids. A general overview of TEAE is displayed in online supplementary Table 1 (see www. karger.com/doi/10.1159/000507381 for all online suppl. material,). Of note, the spectrum of TEAE in TKI and immunotherapy was not overlapping. 


\section{Discussion}

The therapeutic landscape of advanced HCC has profoundly changed over the past years following positive phase III trials [9-12] and promising results from earlierphase clinical trials for immunotherapy $[13,14]$. Treatment allocation remains challenging in daily practice, especially after progression and/or intolerance to first-line systemic therapy with SOR. To our knowledge, our study represents the first analysis of sequential systemic therapy in a real-life cohort of patients with advanced HCC. Our observed median OS of 37.4 months following a median of 3 sequential treatments is unprecedented in advanced HCC so far. Despite this finding, the efficacy of each individual agent in terms of PFS was not higher compared to recent reports $[6,10,13,17]$. In detail, PFS on SOR, REG, and NIVO was $6.6,5.3$, and 6.6 months in our cohort and 5.5, 3.1, and 3.4-4.1 (TTP) months in the respective trials $[6,10,13]$. Considering additional analysis from the RESORCE trial, TTP on SOR was 7.2 months for patients who received subsequent treatment with REG [17], suggesting a selection bias towards better outcome in patients that are eligible for subsequent therapies after initial progression. In the RESORCE trial, the OS for the sequence of SOR and REG was 26 months [17], which was shorter compared to the OS that we observed after a median of 3 sequential systemic treatments. Another study, investigating the sequence of SOR and REG in a Japanese real-world cohort, found similar 1-, 2-, and 3-year survival rates compared to our study with 87.0, 64.2, and $58.4 \%$ (median OS not reached), respectively [18]. Importantly, $46 \%$ of the patients in this study received further systemic treatment (i.e., LEN) [18]. In summary, the published studies together with our data suggest an improved OS for patients eligible for sequential systemic treatments beyond second-line therapies. In our study, the majority of patients had extrahepatic spread at the start of SOR (71\%), which has been suggested as a predictor of better treatment response in a subgroup analysis of the pivotal SHARP trial [6]. In contrast, macrovascular invasion, an indicator for poor prognosis, was less common at start of SOR in our patients; however, its occurrence increased for subsequent treatments with REG or NIVO/PEM and other treatment regimes. Furthermore, all patients receiving sequential treatments had wellcompensated liver function, which is another prognostic marker for a better outcome in HCC [5].

Unfortunately, none of the positive phase III clinical trials were biomarker enriched, except for REACH-2 [12], which further complicates effective treatment allocation. The role of AFP for predicting and monitoring treatment response is still controversially discussed [24]. In our exploratory analysis, changes in AFP levels under treatment correlate with response to therapy, in par- ticular in patients with sustained disease control following immunotherapy. Despite this interesting finding, our cohort is too small to draw any meaningful conclusion on the role of AFP in monitoring treatment response.

We analyzed the safety profile for each major agent as well as the cumulative toxicity of the sequential treatment regimen. As reported $[6,7,10,19,20]$, the TEAE profile of SOR and REG were dominated by HFS reactions with 38 and $30 \%$, respectively. Interestingly, and probably due to a selection of SOR-tolerating patients for treatment with REG, toxicity was rather mild and did not lead to cessation or conversion of the treatment towards immunotherapy. Regarding immunotherapy, immune-related $\mathrm{AE}$ (irAE) can be severe and even fatal [21, 22]. In our cohort, frequency of any-grade irAE was slightly lower compared to data from the CHECKMATE-040 and KEYNOTE-224 trials $[13,14]$; nonetheless, appearance of severe irAE during NIVO or PEM resulted in cessation of treatment in all affected patients. Specifically, short-term recovery of organ inflammation, for example, hepatitis, myositis following immunosuppression with steroids, relapsed after re-induction of immunotherapy, ultimately leading to cessation of immunotherapy. Again, due to the small sample size of these rare patients, a final conclusion needs to be taken with care.

Our study cohort included a broad variety of novel and established agents for the treatment of advanced HCC, reflecting availability of drugs and clinical decision-making in a real-life setting. According to our findings, the exact sequence of agents does not seem to be critical for extensive prolongation of survival, which leaves the question of synergistic effects of combined treatments. We observed a complementary spectrum of side effects in terms of quality between TKI and immunotherapy (online suppl. Table 1), arguing in favor of a safe combinatorial therapeutic approach. To this respect, a promising phase $\mathrm{Ib}$ clinical trial tested the combination of LEN and PEM and achieved an ORR of $45 \%$ in advanced HCC [23]. Similarly, the combination of atezolizumab and bevacizumab achieved an ORR of 34\% and has recently been reported to be superior in terms of OS and PFS compared to SOR in a phase III clinical trial (IMbrave-150, NCT03434379). These results have the potential to dramatically change the treatment landscape of HCC.

In conclusion, our data suggest a safe and efficacious use of the currently available systemic agents. In a selected patient cohort, OS can be profoundly prolonged by a sequential therapeutic approach following first-line treatment with SOR. Future research efforts need to focus on biomarkers predicting treatment response and guiding efficient treatment allocation. Finally, the complementary spectrum of tolerability underlines the significance of the promising combinatorial treatment strategies with targeted agents and immunotherapy. 


\section{Statement of Ethics}

The study was approved by the Ethics Committee of the Ärztekammer Hamburg, Germany (approval No. PV3578, December 2010), and written informed consent was obtained from all participants. The study protocol conformed to the ethical guidelines of the 1975 Declaration of Helsinki as reflected in a priori approval by the institution's human research committee.

\section{Disclosure Statement}

H.W. has received consulting fees from Bayer, Eisai, Roche, Ipsen, BMS, and Lilly. K.S. has advised/consulted for Ipsen and Bayer. All remaining authors declare no conflicts of interest for the submitted work.

\section{Funding Sources}

This work was supported by the Deutsche Leberstiftung (K.S.) and Deutsche Forschungsgemeinschaft (J.F., grant FE1746/1-1).

\section{Author Contributions}

J.F., H.W., and K.S. study design. J.F., K.K., H.I., I.G.I., T.F., J.K., A.L., H.W., and K.S. contribution of patients/data. J.F., H.W., K.S. writing of the manuscript. All authors approved the final version of the manuscript.

\section{References}

1 Bray F, Ferlay J, Soerjomataram I, Siegel RL, Torre LA, Jemal A. Global cancer statistics 2018: GLOBOCAN estimates of incidence and mortality worldwide for 36 cancers in 185 countries. CA Cancer J Clin. 2018 Nov;68(6): 394-424.

2 Marrero JA, Kulik LM, Sirlin CB, Zhu AX, Finn RS, Abecassis MM, et al. Diagnosis, Staging, and Management of Hepatocellular Carcinoma: 2018 Practice Guidance by the American Association for the Study of Liver Diseases. Hepatology. 2018 Aug;68(2):723-50.

3 Galle PR, Forner A, Llovet JM, Mazzaferro V, Piscaglia F, Raoul JL, et al.; European Association for the Study of the Liver. Electronic address: easloffice@easloffice.eu; European Association for the Study of the Liver. Electronic address eee, European Association for the Study of the L. EASL Clinical Practice Guidelines: management of hepatocellular carcinoma. J Hepatol. 2018 Jul;69(1):182-236.

4 Vogel A, Cervantes A, Chau I, Daniele B, Llovet J, Meyer T, et al. Hepatocellular carcinoma: ESMO Clinical Practice Guidelines for diagnosis, treatment and follow-up. Ann Oncol. 2018 Oct 1;29(Suppl 4):iv238-55.

5 Villanueva A. Hepatocellular Carcinoma. N Engl J Med. 2019 Apr;380(15):1450-62.

6 Llovet JM, Ricci S, Mazzaferro V, Hilgard P, Gane E, Blanc JF, et al.; SHARP Investigators Study Group. Sorafenib in advanced hepatocellular carcinoma. N Engl J Med. 2008 Jul; 359(4):378-90.

7 Cheng AL, Kang YK, Chen Z, Tsao CJ, Qin S, Kim JS, et al. Efficacy and safety of sorafenib in patients in the Asia-Pacific region with advanced hepatocellular carcinoma: a phase III randomised, double-blind, placebo-controlled trial. Lancet Oncol. 2009 Jan;10(1):25-34.

8 von Felden J, Schulze K, Gil-Ibanez I, Werner T, Wege H. First- and Second-Line Targeted Systemic Therapy in Hepatocellular Carcinoma-An Update on Patient Selection and Response Evaluation. Diagnostics (Basel). 2016 Nov;6(4):E44.
9 Kudo M, Finn RS, Qin S, Han KH, Ikeda K, Piscaglia $F$, et al. Lenvatinib versus sorafenib in first-line treatment of patients with unresectable hepatocellular carcinoma: a randomised phase 3 non-inferiority trial. Lancet. 2018 Mar;391(10126):1163-73.

10 Bruix J, Qin S, Merle P, Granito A, Huang YH, Bodoky G, et al.; RESORCE Investigators. Regorafenib for patients with hepatocellular carcinoma who progressed on sorafenib treatment (RESORCE): a randomised, double-blind, placebo-controlled, phase 3 trial. Lancet. 2017 Jan;389(10064):56-66.

11 Abou-Alfa GK, Meyer T, Cheng AL, ElKhoueiry AB, Rimassa L, Ryoo BY, et al. Cabozantinib in Patients with Advanced and Progressing Hepatocellular Carcinoma. N Engl J Med. 2018 Jul;379(1):54-63.

12 Zhu AX, Kang Y-K, Yen C-J, Finn RS, Galle PR, Llovet JM, et al. REACH-2: A randomized, double-blind, placebo-controlled phase 3 study of ramucirumab versus placebo as second-line treatment in patients with advanced hepatocellular carcinoma (HCC) and elevated baseline alpha-fetoprotein (AFP) following first-line sorafenib. J Clin Oncol. 2018; 36(15_suppl):4003.

13 El-Khoueiry AB, Sangro B, Yau T, Crocenzi TS, Kudo M, Hsu C, et al. Nivolumab in patients with advanced hepatocellular carcinoma (CheckMate 040): an open-label, noncomparative, phase $1 / 2$ dose escalation and expansion trial. Lancet. 2017 Jun;389(10088): 2492-502.

14 Zhu AX, Finn RS, Edeline J, Cattan S, Ogasawara S, Palmer D, et al.; KEYNOTE-224 investigators. Pembrolizumab in patients with advanced hepatocellular carcinoma previously treated with sorafenib (KEYNOTE-224): a non-randomised, open-label phase 2 trial. Lancet Oncol. 2018 Jul;19(7):940-52.

15 Lencioni R, Llovet JM. Modified RECIST (mRECIST) assessment for hepatocellular carcinoma. Semin Liver Dis. 2010 Feb;30(1): 52-60.
16 von Elm E, Altman DG, Egger M, Pocock SJ, Gøtzsche PC, Vandenbroucke JP; STROBE Initiative. The Strengthening the Reporting of Observational Studies in Epidemiology (STROBE) Statement: guidelines for reporting observational studies. Int J Surg. 2014 Dec;12(12):1495-9.

17 Finn RS, Merle P, Granito A, Huang YH, Bodoky G, Pracht M, et al. Outcomes of sequential treatment with sorafenib followed by regorafenib for HCC: additional analyses from the phase III RESORCE trial. J Hepatol. 2018 Aug;69(2):353-8.

18 Ogasawara S, Ooka Y, Itokawa N, Inoue M, Okabe S, Seki A, et al. Sequential therapy with sorafenib and regorafenib for advanced hepatocellular carcinoma: a multicenter retrospective study in Japan. Invest New Drugs. 2020 Feb;38(1):172-80.

19 Fraile M, Ferrer MT, Matilla AM, Lledó JL, Minguez B, Hernandez A, et al. Efficacy and safety of REGORAFENIB in real life in the treatment of hepatocellular carcinoma. Multicenter experience. J Hepatol. 2018;68:S15.

20 Marrero JA, Kudo M, Venook AP, Ye SL, Bronowicki JP, Chen XP, et al. Observational registry of sorafenib use in clinical practice across Child-Pugh subgroups: the GIDEON study. J Hepatol. 2016 Dec;65(6):1140-7.

21 Postow MA, Sidlow R, Hellmann MD. Immune-Related Adverse Events Associated with Immune Checkpoint Blockade. N Engl J Med. 2018 Jan;378(2):158-68.

22 Wang DY, Salem JE, Cohen JV, Chandra S, Menzer C, Ye F, et al. Fatal Toxic Effects Associated With Immune Checkpoint Inhibitors: A Systematic Review and Meta-analysis. JAMA Oncol. 2018 Dec;4(12):1721-8.

23 Ikeda M, Sung MW, Kudo M, Kobayashi M, Baron AD, Finn RS, et al. A phase $1 \mathrm{~b}$ trial of lenvatinib (LEN) plus pembrolizumab (PEM) in patients (pts) with unresectable hepatocellular carcinoma (uHCC). J Clin Oncolo. 2018; 36(15_suppl):4076. 